\title{
Orientação profissional e vocacional: análise da produção científica
}

\author{
Ana Paula Porto Noronha ${ }^{1}$ \\ Rodolfo Augusto Matteo Ambiel
}

\begin{abstract}
Resumo
O presente estudo objetivou analisar a produção científica brasileira da área de orientação profissional no período compreendido entre a década de 50 até o ano de 2005. Para tanto, procedeu-se à busca bibliográfica nas bases de dados eletrônicas BVS e IndexPsi em fevereiro de 2006, utilizando-se as palavras-chave Orientação Profissional, Orientação Vocacional, Interesses Profissionais, Escolha Profissional e Testes de Interesse para busca. Dessa busca, foram extraídos resumos e referências, daqueles que os possuíam. Os resultados revelaram que houve um aumento da produção a partir da década de 90 e que os trabalhos tratavam mais de revisões teóricas e de verificação da qualidade dos instrumentos de avaliação. Dentre as estratégias de avaliação mais utilizadas encontravam-se as técnicas não padronizadas e os instrumentos de interesse.

Palavras-chave: Avaliação psicológica; Testes psicológicos; Testes de interesse.
\end{abstract}

\section{Professional and vocacional counseling: Scientific production analysis}

\begin{abstract}
This study aimed to analyse the brazilian scientific production in the professional counseling area from 1950 decade to 2005. For such, the authors proceeded the search in the BVS and IndexPsi electronic database in February, 2006, using the keywords Professional Counseling, Vocacional Counseling, Professional Insterests, Professional Choice and Interests tests. From this search, references and abstracts were extracted, from those that offered it. The results revealed that there were a increasing in the production since 1990 decade and that the works studied about theoretical review and the quality of assessment instruments. Among the most used evaluation strategies were found the interviews and other and the interests tests.

Keywords: Psychological assessment; Psychological tests; Interests tests.
\end{abstract}

\section{Introdução}

A prática profissional pode ser considerada como uma das atividades de maior importância na vida de um indivíduo adulto, sendo que é na adolescência, fase na qual se intensificam as dúvidas a respeito do futuro, que os interesses profissionais começam a evidenciar-se, tendendo a se resolver até o início da vida adulta (Leitão \& Miguel, 2004). Nesse processo de escolha profissional, questões como identificações, aptidões, situações familiares e perspectivas para o futuro são importantes elementos de investigação (Primi, Casellato \& Moggi, 2005).

Sob essa perspectiva, a Psicologia oferece seus instrumentos, visando proporcionar reflexão e autoconhecimento por meio da Orientação Profissional (OP). Para Bock e colaboradores (1995) a função do psicólogo é promover a saúde, e de alguma forma, o trabalho de OP pretende atingir tal objetivo, já que leva o sujeito a se conhecer, no sentido de possibilitar uma escolha mais lúcida, madura, ajustada e de acordo com as habi- lidades de cada indivíduo. Ao lado disso, segundo Anastasi e Urbina (2000), os inventários de interesses profissionais têm sido usados também para familiarizar o indivíduo com as opções adequadas de ocupações, com as quais não teria contato de outra forma.

Apesar da visão clínico-escolar que parece acompanhar seu desenvolvimento (Angelini, 1954; Ribeiro \& Bessa, 1960; Aguiar \& cols., 1978; Kessler \& cols., 1983; Oliveira, 1995), o campo de ação da OP excede esses limites e se projeta para um lugar de destaque no âmbito da psicologia organizacional, como afirmam Anastasi e Urbina (2000). As autoras enfatizam que os testes são comumente usados nesses contextos como auxílio nas decisões ocupacionais relativas à seleção e à classificação de pessoal. Assim, vários trabalhos brasileiros fazem alusão ao desenvolvimento do uso de instrumentos de OP em empresas ou em cursos profissionalizantes. Na década de 50, Barioni e Jorge (1952) já relatavam o uso de diversos instrumentos no Departamento Regional de São Paulo do SENAC, visando uma melhor adaptação dos menores que se formavam em

\footnotetext{
${ }^{1}$ Endereço para correspondência:

Rua Alexandre Rodrigues Barbosa, 45, Centro - 13251-900 - Itatiba-SP

E-mail: ana.noronha@saofrancisco.edu.br
} 
seus cursos nos cargos oferecidos pelas empresas. Ao lado disso, Santos (1967) relata experiência referente à colocação profissional de deficientes visuais na indústria, por meio do SENAI. Sparta (2003), por sua vez, descreveu o desenvolvimento da Orientação Profissional brasileira. E, nesse sentido, destaque pode ser dado às relações entre a OP e aos principais modelos teóricos sobre escolha profissional utilizados em nosso meio.

Os instrumentos de avaliação de interesses profissionais, embora constituam parte importante do processo, não devem ser tomados como um fim. A American Psychological Association, (APA, 1985) incentiva em seu Standards for Educational and Psychological Testing o uso de outros materiais, técnicas e informações juntamente com os resultados dos testes, para que sejam feitas previsões e tomadas decisões no âmbito ocupacional, reforçando a tese de que, dentre as alternativas de avaliação disponíveis, os testes, quando utilizados adequadamente, são as soluções mais válidas e menos discriminatórias.

Exemplos de avaliação utilizando-se de várias técnicas são encontrados desde as primeiras experiências de OP relatadas no Brasil. Barioni e Jorge (1952) relatam que em seu trabalho de orientação no SENAC, provas de inteligência (Prova de Maia e Prova de Mieli), memória (não citadas) e personalidade (Rorschach, Miocinético de Mira, Catálogo de Livros, entre outros) eram aplicadas buscando obter escores que pudessem indicar a melhor posição a ser ocupada. A avaliação era conduzida por uma equipe multidisciplinar, formada por funcionários do departamento de OP, orientadores educacionais e médicos. Larqué (1980) apresenta estudo, fruto da parceria do Instituto de Seleção e Orientação Profissional (ISOP) com o Ministério da Educação (MEC), desenvolvido em 77 cidades brasileiras, com uma amostra total de 79.569 alunos de escolas públicas e particulares, no qual há o uso de testes objetivos de rendimento escolar juntamente com testes de aptidões diversificadas, tais como raciocínio verbal, numérico e mecânico, desenvolvidos especialmente para a situação. O estudo teve como objetivo principal fornecer para as escolas subsídios para a implantação de novos cursos profissionalizantes no $2^{\circ}$ grau.

Angelini e Agatti (1984) procuraram estabelecer as relações entre aptidão intelectual e as discrepâncias entre variáveis relativas às aspirações e expectativas profissionais, tanto dos pais quanto dos adolescentes, por meio do Teste Matrizes Progressivas de Raven e de um Inventário de Interesses Profissionais do primeiro autor, concluindo que a orientação profissional deve também apresentar uma face familiar, buscando produzir atitudes mais realistas diante da escolha profissional. Mais recentemente, Bueno, Lemos e Tomé (2004) buscaram corre- lacionar os interesses profissionais com inteligência e personalidade em um grupo de estudantes de psicologia por meio do Levantamento de Interesses Profissionais (LIP), Matrizes Progressivas de Raven e o Inventário de 16 Fatores de Personalidade (16-PF). Os resultados levaram os autores a afirmar que há relação entre essas características e as diferentes possibilidades de atuação do psicólogo.

No sentido da readaptação profissional, alguns estudos foram realizados desde a década de 60 , tal como o de Mignolet (1960) que, fazendo uma revisão sobre o desenvolvimento senil e o envelhecimento da população e suas conseqüências, procede uma reflexão sobre a utilização adequada das técnicas e métodos de OP para a readaptação e reeducação profissional de pessoas idosas. Silva (2001) relata a crescente procura de adultos jovens pela reorientação profissional e sinaliza a necessidade de se abordar o problema de acordo com as características desse novo tipo de cliente e a produção de conhecimento para que os orientadores possam trabalhar com segurança com essa nova clientela. Outros trabalhos nesse sentido também foram realizados, tais como o de Silva (1966), Scheefer (1973) e Soares-Lucchiari (1997). Por outro lado, Pasqualini, Garbulho e Schut (2004), com base na perspectiva sócio-histórica, trazem uma experiência de OP com crianças pré-escolares, defendendo que nessa faixa etária as crianças começam a reproduzir as funções sociais dos adultos, e assim, a orientação nessa idade favoreceria uma formação integral do indivíduo, podendo o psicólogo encontrar aí um futuro campo de atuação.

Para uma maior integração entre as várias facetas do mundo do trabalho com os conhecimentos já produzidos pela psicologia, é importante que cada vez mais se produza ciência voltada a essa intersecção, visando em última instância proporcionar melhor qualidade de vida para aqueles que se beneficiam dessa importante interface. Nesse sentido, Ottati, Noronha e Salviati (2003) e Noronha, Freitas e Ottati (2003) apresentam trabalhos analisando a qualidade dos instrumentos de avaliação de interesses profissionais publicados no Brasil. Num período em que as resoluções do CFP (2001, 2003) concernentes às propriedades psicométricas exigidas dos instrumentos ainda estavam provocando os primeiros efeitos, os autores constataram que a qualidade não era satisfatória em vários aspectos, desde a falta de estudos científicos até a má qualidade dos manuais técnicos, que deixavam de apresentar informações importantes sobre as características e normas de aplicação e correção, fatos que indicavam a necessidade de mais estudos que possibilitassem o uso desses instrumentos nos processos de OP.

No que se refere ao tema, a psicologia brasileira ainda se mostra incipiente com relação aos instrumen- 
tos facilitadores da escolha profissional, o que sugere urgência no desenvolvimento de pesquisas. A esse respeito vale destacar que, dentre os instrumentos psicológicos aprovados pelo CFP, encontra-se uma predominância daqueles que objetivam avaliar inteligência $\mathrm{e}$ personalidade, enquanto que instrumentos específicos para avaliação em situações de orientação profissional ainda são escassos no contexto nacional (CFP, 2005). Nada obstante, os construtos mais pesquisados parecem ser convergentes com a proposta da OP, já que a avaliação de aptidões e características de personalidade permeiam o processo e parecem ser critérios importantes na escolha e vínculo a uma determinada carreira (Primi, Moggi \& Casellato, 2004).

Além das questões já apontadas, o exercício profissional da OP vem se deparando com alguns paradoxos. Lisboa (2002) afirma que a formação dos orientadores vem se desenvolvendo de forma importante, porém lenta, no sentido de que os cursos universitários que formam os orientadores dentro de suas especialidades, a saber, Psicologia e Pedagogia, não dão ainda a importância devida a esse campo de conhecimento. Como conseqüência, a atuação tende a ser fragmentada (Lehman, 1988; Bueno, Lemos \& Tomé, 2004; Nascimento, 2004).

Oliveira (1999) indica um movimento ambíguo de carência do novo e nostalgia do antigo, no qual as técnicas de OP são aplicadas sem a devida reflexão, o que causa dificuldades no desenvolvimento do processo perante as várias mudanças nos setores que exercem influência e que são influenciados pelas questões relativas ao trabalho. Também sob essa perspectiva, Silva (1999) enfatiza que a formação dos orientadores profissionais deve estar baseada em três pilares, a saber, base teórica e técnica consistente, clareza na visão de homem e de mundo e resolução dos próprios conflitos quanto à escolha; a idéia é corroborada por Nickel (1996), ao afirmar que características pessoais do orientador, consoante à sua formação, influenciam todo o processo de escolha do orientando.

Percebe-se que as várias possibilidades teóricas existentes na Psicologia vêm se debruçando sobre as questões da OP, realçando as diferentes formas de atuação. Exemplo disso são os vários estudos realizados com o Teste de Fotos de Profissões (BBT), uma técnica projetiva específica para avaliação em contexto de OP que, não obstante, é o único instrumento atualmente aprovado pelo CFP que visa de fato facilitar a escolha da profissão (Jacquemin, 1982; Melo-Silva \& Santos, 1998; Silva \& Jacquemin, 2000; Magalhães, 2002; Okino e cols., 2003). Canedo (1997), por outro lado, faz uma análise da relação existente entre os pressupostos da Gestalt-terapia com as propostas clínicas de Bohos- lavsky (1993). Gimenez (1998) também se baseia no autor argentino e acrescenta ao seu trabalho de OP o Sandplay e análise de sonhos, próprios da prática clínica. Buscando posicionar a atuação do orientador profissional na clínica psicanalítica, Velloso (2000) situa o fenômeno da adolescência na teoria freudiana, propondo uma modalidade clínica na qual mescla os conhecimentos da psicologia e da psicanálise. Por sua vez, Moura e Silveira (2002) enfocam a OP no âmbito da análise do comportamento, buscando identificar e avaliar as mudanças comportamentais de adolescentes quanto aos indicadores de escolha profissional.

Como se pode perceber, a OP tem grande importância para a psicologia brasileira. Nesse sentido, a análise da produção científica da área se faz necessário, uma vez que seus resultados poderão nortear ações futuras de pesquisas e intervenções. Para Witter (1999), o progresso da ciência está intrinsecamente relacionado com a avaliação de suas publicações e pode ser realizado por meio da compreensão da temática, do discurso, da metodologia, do procedimento de análise de dados e dos variados enfoques teóricos.

Nesse sentido, o estudo de Melo-Silva (1999) visou investigar o estado da arte do domínio da orientação profissional brasileira. Seus achados revelaram que a partir da década de 90 houve um aumento das produções, e que os trabalhos que tratavam de intervenção foram os mais freqüentes. Tendo isso em vista, o presente trabalho pretendeu analisar a produção científica brasileira na área de orientação profissional no período compreendido entre 1950 e 2005.

\section{Método}

\section{Material e procedimento}

Foi procedida a busca bibliográfica por publicações brasileiras na área de OP nas bases de dados eletrônicas BVS e IndexPsi em fevereiro de 2006, utilizando-se os termos Orientação Profissional, Orientaşão Vocacional, Interesses Profissionais, Escolha Profissional e Testes de Interesse como chave para busca. Dessa busca, foram extraídos os resumos e referência daqueles que os tinham disponíveis e apenas as referências daqueles que contavam com os resumos.

Inicialmente, tinha-se a intenção de buscar aqueles publicados após 1960, mas com a pesquisa percebeu-se a necessidade de aumentar essa margem de tempo em mais uma década, já que foram encontradas publicações também na década de 1950. Anterior a essa data, nenhuma publicação foi encontrada. Ainda, op tou-se apenas por artigos publicados em revista científicas da área de psicologia, já que na base BVS foram 
Tabela 1 - Freqüência e porcentagem da produção por década

\begin{tabular}{cccc}
\hline Década & Freqüência & $\%$ & $\%$ acumulada \\
\hline 1950 & 26 & 13,6 & 13,6 \\
1960 & 32 & 16,8 & 30,4 \\
1970 & 15 & 7,9 & 38,2 \\
1980 & 22 & 11,5 & 49,7 \\
1990 & 46 & 24,1 & 73,8 \\
2000 & 50 & 26,2 & 100,0 \\
\hline Total & 191 & 100,0 & \\
\hline
\end{tabular}

encontrados artigos que tratavam de orientação para o trabalho, mas voltadas e publicadas em revistas de outras especialidades da área de saúde, tais como Enfermagem, Medicina e Odontologia. Com isso, dissertações de mestrado, teses de doutorado e capítulos de livros concernentes ao assunto e não publicados também foram desconsiderados.

Após isso, procedeu-se à análise dos resumos disponíveis a fim de extrair as informações que interessavam para compor o estudo. Nessas análises, buscouse identificar o tipo do artigo (pesquisa, teórico, relatos de experiência ou comunicação); os temas dos artigos (Qualidade do Processo, Qualidade da Técnica, Qualidade do Instrumento, OP voltada para público específico, Histórico, Revisão Teórica e Caracterização) e informações a respeito da técnica ou instrumento utilizado; além da caracterização da amostra pesquisada. As sete categorias de temas foram compostas por meio de uma análise de conteúdo dos resumos ou dos artigos, buscando identificar as características em comum entre eles, tomado-se como base os pressupostos de Bardin (1977).

\section{Resultados}

A busca na base de dados permitiu recuperar 191 artigos com as palavras-chave descritas no método. Desses, aproximadamente $50 \%$ foram produzidos até a década de 80, e a partir da década de 1990 nota-se crescimento da área, conforme apresentado na Tabela 1.
O número de autores dos manuscritos também foi investigado e pode ser visualizado na Tabela 2. Os resultados permitem compreender que a maior parte dos estudos é elaborada por um único autor e que apenas uma pequena parte é fruto de trabalhos de três autores ou mais.

A Tabela 3 apresenta os dados relativos aos periódicos nos quais os manuscritos foram publicados. Como se pode observar, o maior número de artigos está nos Arquivos Brasileiros de Psicotécnica e na Revista da $A B O P$, considerando as palavras-chave em questão. Quanto à primeira revista, tal condição se deve ao fato de que a mesma monipolizou as publicações durante as décadas de 1950 e 1960. Já a segunda, por se tratar de um periódico especializado na área, apresentou uma produção expressiva, especialmente na década de 1990.

Para as análises seguintes serão considerados os artigos, cujos resumos estavam disponíveis nas respectivas bases de dados consultadas. Assim, o N total não será mais 191. O tipo de investigação realizada foi observado, sendo que para essa análise foram definidas quatro categorias, quais sejam, artigos de pesquisa, artigos teóricos, relatos de experiência e comunicações orais. Os artigos de pesquisa e os teóricos representam a grande maioria dos tipos de publicações, o que pode ser explicado em razão dos periódicos consultados, quais sejam, os que se relacionam mais diretamente com trabalhos dessa natureza.

Tabela 2 - Freqüência e porcentagem do número de autores

\begin{tabular}{cccccc}
\hline & Freqüência & $\%$ & $\%$ válida & \% acumulada \\
\hline & 1 & 126 & 66,0 & 66,7 & 66,7 \\
& 2 & 36 & 18,8 & 19,0 & 85,7 \\
& 3 & 11 & 5,8 & 5,8 & 91,5 \\
& 4 & 7 & 3,7 & 3,7 & 95,2 \\
& 5 & 5 & 2,6 & 2,6 & 97,9 \\
& 6 & 3 & 1,6 & 1,6 & 100,0 \\
& 13 & 1 & 0,5 & 0,5 & \\
& Total & 189 & 99,0 & 100,0 & \\
Perda & 2 & 1,0 & Psico-USF, v. 11, n. 1, p. . 75-84, jan. /jun. 2006 \\
Total & 191 & 100,0 & & \\
\hline
\end{tabular}


Tabela 3 - Freqüência e porcentagem dos periódicos

\begin{tabular}{|c|c|c|}
\hline & Freqüência & $\%$ \\
\hline Arquivos Brasileiros de Psicotécnica & 51 & 26,7 \\
\hline Boletim de Psicologia & 11 & 5,8 \\
\hline Revista de Psicologia Normal e Patológica & 1 & 0,5 \\
\hline Jornal Brasileiro de Psicologia & 1 & 0,5 \\
\hline Arquivo Brasileiro de Psicologia Aplicada & 14 & 7,3 \\
\hline Arquivo Brasileiro de Psicologia & 14 & 7,3 \\
\hline Psicologia em Curso & 1 & 0,5 \\
\hline Psico (Porto Alegre) & 6 & 3,1 \\
\hline Cadernos de Psicologia (Belo Horizonte) & 4 & 2,1 \\
\hline Psicologia Teoria e Pesquisa & 3 & 1,6 \\
\hline Revista de Psicologia & 2 & 1,0 \\
\hline Perfil: Boletim de Psicologia & 1 & 0,5 \\
\hline Revista Brasileira de Pesquisa em Psicologia & 1 & 0,5 \\
\hline Estudos de Psicologia (Campinas) & 4 & 2,1 \\
\hline Doxa & 3 & 1,6 \\
\hline Psicologia Argumento & 7 & 3,7 \\
\hline Revista Brasileira de Estudos Pedagógicos & 1 & 0,5 \\
\hline Revista da ABOP & 23 & 12,0 \\
\hline Alethéia & 4 & 2,1 \\
\hline Psicologia: Ciência e Profissão & 2 & 1,0 \\
\hline Junguiana & 1 & 0,5 \\
\hline Psicologia Escolar e Educacional & 3 & 1,6 \\
\hline Interações Estudos e Pesquisa em Psicologia & 1 & 0,5 \\
\hline Psic & 6 & 3,1 \\
\hline Psicologia Reflexão e Crítica & 1 & 0,5 \\
\hline Cogito & 1 & 0,5 \\
\hline Psicologia em Estudo & 4 & 2,1 \\
\hline Psicologia Revista & 2 & 1,0 \\
\hline Estudos de Psicologia (Natal) & 1 & 0,5 \\
\hline Adolescente Latino-Americano & 1 & 0,5 \\
\hline Revista Brasileira de Orientação Profissional & 13 & 6,8 \\
\hline Interação (Curitiba) & 1 & 0,5 \\
\hline Psicologia Educacional & 1 & 0,5 \\
\hline Barbarói & 1 & 0,5 \\
\hline Total & 191 & 100,0 \\
\hline
\end{tabular}

Para a análise dos temas tratados nos estudos, sete categorias foram definidas pelos pesquisadores, e os artigos foram classificados por eles, que funcionaram como juízes independentes. A concordância foi de $80 \%$. Os resultados podem ser observados na Tabela 5 .

Para compreensão dos temas tratados foram es- tabelecidas 7 categorias de análise, sendo que em Qualidade do Processo foram incluídos os trabalhos que tinham como objetivo investigar a eficácia do processo de OP (Melo-Silva, Oliveira \& Coelho, 2002); por Qualidade da Técnica e Qualidade do Instrumento entendeu-se os estudos que visavam verificar se as diferentes técnicas e/ou

Tabela 4 - Freqüência e porcentagem dos tipos de publicações

\begin{tabular}{lccc}
\hline & Freqüêencia & $\%$ & $\%$ acumulado \\
\hline Artigos de pesquisa & 52 & 27,2 & 39,1 \\
Artigos teóricos & 55 & 28,8 & 80,5 \\
Relatos de experiência & 20 & 10,5 & 95,5 \\
Comunicação oral & 6 & 3,1 & 100,0 \\
\hline Total & 133 & 69,6 &
\end{tabular}

Psico-USF, v. 11, n. 1, p. 75-84, jan./jun. 2006 
Tabela 5 - Freqüência e porcentagem dos temas dos artigos consultados

\begin{tabular}{lccc}
\hline Temas & Freqüência & $\%$ & $\%$ acumulada \\
\hline Qualidade do processo & 23 & 17,3 & 17,3 \\
Qualidade da técnica & 11 & 8,3 & 25,6 \\
Qualidade do instrumento & 30 & 22,5 & 48,1 \\
OP voltada para público específico & 9 & 6,7 & 54,8 \\
Histórico & 5 & 3,7 & 58,5 \\
Revisão teórica & 44 & 33,2 & 91,7 \\
Caracterização & 11 & 8,3 & 100,0 \\
\hline Total & 133 & 70,2 & \\
\hline
\end{tabular}

instrumentos utilizados contribuíam com informações importantes para a coleta de dados (Moura, Sampaio, Menezes \& Rodrigues, 2003; Silva \& Jacquemin, 2000). $\mathrm{Na}$ categoria OP voltada para público especifico foram incluídos os argumentos relativos aos trabalhos desenvolvidos com grupos, tais como deficientes visuais (Santos, 1966) ou pessoas em situações específicas, tal como a reorientação profissional (Moura \& Menezes, 2004). O Histórico tratou de revisões históricas da orientação profissional (Rapold, 2003), enquanto a Revisão Teórica, da discussão de modelos teóricos (Canedo, 1997). Por fim, a Caracterização envolveu os trabalhos que abordaram a realização de estudos que buscavam caracterizar populações específicas com relação a variáveis referentes aos interesses ou processos de escolha profissional (Bueno, Lemos \& Tomé, 2004).

Ainda no que diz respeito à análise dos resumos, os instrumentos utilizados foram reunidos em 8 categorias de análise, quais sejam, Testes de Inteligência e Aptidão (exemplo: Raven, D48), Testes de Personalidade (Rorschach, IFP), Testes de Interesses (BBT, SDS), Testes de Maturidade ou Desenvolvimento Profissional (EMEP), Outros Testes (Inventário de Papéis Sexuais de Bem), Técnicas (Entrevista), Técnicas Alternativas (cartazes, colagem) e Outros Não-Identificados (Q-Sorte). A classificação dos instrumentos se deu de maneira independente pelos dois autores, sendo que a concordância entre eles foi de 90\% (Tabela 6).
Dos 133 artigos que contavam com resumos disponíveis, apenas 60 relatavam o uso de instrumentos ou técnicas. Nesses relatos, foram encontrados 80 instrumentos usados, já que os estudos variavam entre o mínimo de um e o máximo de três instrumentos e/ou técnica. Destaque deve ser dado ao uso de Ténnicas, tais como entrevistas e questionários e Testes de Interesses. Além desses, o uso de instrumentos que se prestam a avaliar maturidade para escolha, desenvolvimento profissional, personalidade e inteligência/aptidão também foram utilizados (Tabela 6).

Ao se comparar a década de publicação com o tipo de trabalho, observou-se uma predominância de artigos de pesquisa, especialmente de 2000 a 2005. Também nesse sentido, há um aumento do número de trabalhos ao longo das décadas, como demonstra a Tabela 7.

Já no tocante à análise da distribuição dos temas dos trabalhos pelas décadas investigadas, observouse que, dentre as sete categorias encontradas, houve uma predominância de Revisões Teóricas, que envolveram estudos teóricos com variadas abordagens de OP. Os dados podem ser encontrados na Tabela 8 .

Ao lado disso, os temas relacionados à Qualidade de Instrumento e Qualidade de Processo também foram bem representados. Nesse sentido, o primeiro tema engloba os estudos que visaram atestar a qualidade técnica de algum instrumento de avaliação psicológica para a área de OP. E, nessa direção, vale lembrar que são usados

Tabela 6 - Freqüência dos tipos de testes

\begin{tabular}{lcc}
\hline & Freqüência & $\%$ \\
\hline Testes de inteligência e aptidão & 8 & 10,0 \\
Testes de personalidade & 10 & 12,5 \\
Testes de interesses & 18 & 22,5 \\
Testes de maturidade ou desenvolvimento profissional & 9 & 11,3 \\
Outros testes & 7 & 8,8 \\
Técnicas & 19 & 23,8 \\
Técnicas alternativas & 7 & 8,8 \\
Outros não-identificados & 2 & 2,5 \\
\hline Total & 80 & 100,0 \\
\hline
\end{tabular}


Tabela 7 - Freqüência dos tipos de publicação por década

\begin{tabular}{llllllll}
\hline \multicolumn{7}{c}{ Década } \\
\hline Tipos & 1950 & 1960 & 1970 & 1980 & 1990 & 2000 & Total \\
\hline Pesquisa & 0 & 4 & 4 & 8 & 6 & 30 & 52 \\
Téorico & 3 & 0 & 4 & 10 & 22 & 16 & 55 \\
Relato de experiência & 2 & 0 & 2 & 2 & 11 & 3 & 20 \\
Comunicação oral & 0 & 1 & 3 & 1 & 0 & 1 & 6 \\
\hline Total & 5 & 5 & 13 & 21 & 39 & 50 & 133 \\
\hline
\end{tabular}

instrumentos que se destinam a avaliar construtos distintos, tais como inteligência e personalidade.

A categoria Técnicas, que engloba questionários, entrevistas semi ou não-estruturadas e demais instrumentos não padronizados, foi a que obteve maior freqüência (Tabela 9). Nessa categoria, percebeu-se um grande número de técnicas desenvolvidas para atender aos propósitos exclusivos da pesquisa, não mais sendo utilizadas em outros estudos.

Os testes de interesse, de maturidade ou desenvolvimento profissional e os de personalidade apresentaram-se numa quantidade importante, evidenciando a relevância deles. Também nessa categoria notou-se uma variedade de instrumentos utilizados em único estudo.

\section{Discussão e considerações finais}

O presente estudo objetivou estudar a produção científica da área de orientação profissional por meio da análise dos trabalhos encontrados nas bases de dados BVS e IndexPsi, meio eletrônico. Os resultados permitiram algumas reflexões que se seguem.

Embora tenha sido encontrada produção sobre o tema desde 1950 até os dias atuais, é importante notar que a partir da década de 90 houve um crescimento da divulgação de artigos teóricos e de pesquisas, bem como de trabalhos de outra natureza, como já evidenciado nos achados de Melo-Silva (1999). Tal fato pode estar relacionado com a relevância da orientação para o processo de escolha do jovem (Primi, Casellato \& Moggi, 2005; Bueno, Lemos \& Tomé, 2004; Leitão \& Miguel, 2004; Nascimento, 2004; Silva, 2001; Lehman, 1988), ao mesmo tempo que pode estar em consonância com as discussões sobre a qualidade dos instrumentos de avaliação (Ottati, Noronha \& Salviati, 2003; Noronha, Freitas \& Ottati, 2003) e com a preocupação acerca da formação profissional do orientador (Nickel, 1996).

Em outra medida, a maior parte dos trabalhos consultados foi escrita por um único autor, revelando a produção prioritariamente solitária do pesquisador brasileiro. Já no que se refere ao tipo de publicação, os dados revelaram que os teóricos e os de pesquisa foram os predominantes, o que era previsível, considerando a especificidade dos periódicos consultados, como já abordado quando da apresentação dos resultados. Também nessa direção, qual seja, dos periódicos, alguns deles se destacaram em relação ao número de publicações em razão da especificidade de suas publicações e da falta de opções em tempos mais remotos.

Dentre os temas categorizados para o presente estudo, a preocupação com os instrumentos esteve mais representado, mais especialmente no que respeita à qualidade dos instrumentos utilizados no processo de orientação profissional. Essa é uma tendência contemporânea da avaliação psicológica brasileira, sobretudo após as resoluções promulgadas pelo Conselho Federal de Psicologia, a fim de regulamentar o uso e a construção de instrumentos de medida (CFP, 2001, 2003; Anastasi \& Urbina, 2000).

Os instrumentos mais utilizados nos estudos foram o Kuder Preference Record e o BBT. O primeiro se apresentou principalmente em estudos das décadas de 60 e 70, enquanto que o segundo foi encontrado em vários estudos ao longo do período analisado, mas principalmente nas décadas de 1990 e 2000 . Não por acaso,

Tabela 8 - Freqüência dos temas das publicações por década

\begin{tabular}{|c|c|c|c|c|c|c|c|}
\hline \multirow[t]{2}{*}{ Temas } & \multicolumn{7}{|c|}{ Década } \\
\hline & 1950 & 1960 & 1970 & 1980 & 1990 & 2000 & total \\
\hline Qualidade de processo & 2 & 1 & 1 & 6 & 6 & 7 & 23 \\
\hline Qualidade de técnica & 1 & 0 & 1 & 1 & 6 & 2 & 11 \\
\hline Qualidade de instrumento & 1 & 4 & 2 & 7 & 4 & 12 & 30 \\
\hline OP Voltada para público específico & 0 & 2 & 1 & 0 & 1 & 5 & 9 \\
\hline Históricos & 0 & 0 & 3 & 0 & 0 & 2 & 5 \\
\hline Revisão teórica & 1 & 0 & 5 & 7 & 21 & 10 & 44 \\
\hline Caracterização & 0 & 0 & 0 & 0 & 1 & 10 & 11 \\
\hline Total & 5 & 7 & 14 & 21 & 39 & 48 & 133 \\
\hline
\end{tabular}

Psico-USF, v. 11, n. 1, p. 75-84, jan./jun. 2006 
Tabela 9 - Freqüência dos tipos de instrumentos por década

\begin{tabular}{lccccccc}
\hline \multirow{2}{*}{ Tipos de Instrumentos } & \multicolumn{7}{c}{ Década } \\
\cline { 2 - 8 } \multicolumn{1}{c}{} & 1950 & 1960 & 1970 & 1980 & 1990 & 2000 & Total \\
\hline Testes de inteligência e aptidão & 0 & 0 & 0 & 4 & 0 & 4 & 8 \\
Testes de personalidade & 1 & 1 & 1 & 0 & 2 & 5 & 10 \\
estes de interesses & 0 & 5 & 1 & 3 & 3 & 6 & 18 \\
Testes de maturidade ou desenvol. profissional & 0 & 0 & 0 & 2 & 0 & 7 & 9 \\
Outros testes & 0 & 1 & 1 & 2 & 1 & 2 & 7 \\
Técnicas & 0 & 1 & 0 & 0 & 3 & 15 & 19 \\
Técnicas alternativas & 1 & 0 & 0 & 0 & 2 & 4 & 7 \\
Outros não identificados & 0 & 1 & 0 & 0 & 1 & 0 & 2 \\
\hline \multicolumn{1}{c}{ Total } & 2 & 9 & 3 & 11 & 12 & 43 & 80 \\
\hline \multicolumn{1}{c}{} & & & & & & &
\end{tabular}

o BBT é o único instrumento que se encontra aprovado pelo CFP para comercialização e uso no Brasil, como o objetivo de avaliar inclinações profissionais.

A análise do tipo de publicação por década permitiu identificar um aumento da produção a cada período, o que pode ser um sinal importante de crescimento da área, à medida que pode reafirmar que ela é promissora. Nesse sentido, as asserções de Witter (1999) são relevantes. A autora afirma que a investigação sobre o "estado da arte" de determinada área de conhecimento pode auxiliar na definição de metas e de futuras pesquisas. Sob essa perspectiva, a orientação profissional com populações específicas mereceria maior atenção por parte dos pesquisadores.

Quanto aos instrumentos utilizados nos estudos, parece que ainda há muito a desenvolver. Os resultados revelaram que de uma forma geral, vários construtos imbricados no processo de OP merecem um olhar mais refinado, o que possibilita a abertura para novas investigações.

\section{Referências}

Aguiar, F., Muller, H., Meirelles Filho, J., Fontes, I. \& Vaz, S. (1978). Orientação clínico-vocacional. Arquivos Brasileiros de Psicologia Aplicada, 30(1/2), 157-161.

Anastasi, A. \& Urbina, S. (2000). Testagem psicológica. Porto Alegre: Artes Médicas.

Angelini, A. L. (1954). Quais os cursos preferidos pelos alunos dos nossos ginásios? Arquivos Brasileiros de Psicotécnica, 6(3), 11-25.

Angelini, A. L. \& Agatti, A. P. R. (1954). Interesses profissionais e aptidão intellectual. Arquivos Brasileiros de Psicologia, 36(1), 80-88.

Bardin, L. (1970). Análise de conteúdo. Lisboa: Edições 70.

Barioni, W. \& Jorge, B. H. (1952). A orientação profissional no Departamento Regional de S. Paulo do Senac. Boletim de Psicologia, 3/4(11/13), 17-24.
Bock, A M. M. \& colaboradores (1995). A escolha profissional em questão. São Paulo: Casa do Psicólogo.

Bohoslavsky, R. (1993). Orientação vocacional: a estratégia clínica. São Paulo: Martins Fontes.

Bueno, J. M. H., Lemos, C. G., Tomé, F. A. M. F. (2004). Interesses profissionais de um grupo de estudantes de psicologia e suas relações com inteligência e personalidade. Psicologia em estudo, 9(2), 271-278.

Canedo, I. R. (1997). Contribuições da Gestalt-terapia para o referencial teórico da orientação profissional. Revista da ABOP, 1(1), 59-67.

Conselho Federal de Psicologia - CFP (2001). Resolução $\mathrm{n}^{\mathrm{o}}$ 25/2001. Disponível em: <http://www.pol.org.br >. Acesso em: 15/8/2005.

Conselho Federal de Psicologia - CFP (2003). Resolução $\mathrm{n}^{\circ}$ 02/2003. Disponível em: <http://wmw.pol.org.br >. Acesso em: 2/11/2003.

Conselho Federal de Psicologia - CFP (2005). Sistema de Avaliação de Testes psicológicos. Disponível em: <http:/ / www.pol.org.br>. Acesso em: 17/9/2005.

Conselho Federal de Psicologia - CFP (2006). Sistema de Avaliação de Testes psicológicos. Disponível em: <http:/ / www.pol.org.br>. Acesso em: 27/3/06.

Gimenez, P. (1998). Orientação profissional: criação de um espaço ritual através do Sandplay. Junguiana, 16, $55-65$.

Jacquemin, A. (1982). Novas perspectivas em orientação vocacional e profissional. Arquivos Brasileiros de Psicologia, 34(4), 127-134.

Kessler, A. S., Fattori, E. G., Macedo, L. R. \& Silva, R. N. (1983). A orientação vocacional a partir de um enfoque clínico. Psico, 6(1).

Larqué, M. L. (1980). Testes de rendimento na sondagem de aptidões e orientação educacional do aluno. Arquivos Brasileiros de Psicologia, 32(1), 239-249. 
Lehman, Y. P. (1988). Aquisição de identidade vocacional em uma sociedade em crise: dois momentos na profissão liberal. (Tese de doutorado não-publicada). São Paulo: Universidade de São Paulo. 328 p.

Leitão, L. M. \& Miguel, J. P. (2004) Avaliação dos interesses. Em: L. M. Leitão (Org.). Avaliação psicológica em orientação escolar e profissional (pp. 179-262). Coimbra: Quarteto.

Lisboa, M. D. (2002). Orientação profissional e mundo do trabalho: reflexões sobre uma nova proposta frente a um novo cenário. Em R. S. Levenfus \& D. H. P. Soares (orgs.) Orientação vocacional ocupacional: novos achados teóricos, técnicos e instrumentais para a clínica, a escola e a empresa. Porto Alegre: Art Med.

Magalhães, M. O. (2002). Uma introdução ao BBT: um método projetivo para a clarificação da inclinação profissional. Aletheia, 15, 103-110.

Melo-Silva, L. L., Oliveira, J. C. \& Coelho, R. S. (2002). Avaliação da orientação profissional no desenvolvimento da maturidade na escolha da profissão. Psic, 3(2), 44-53.

Melo-Silva, L. L. \& Santos, M. A. (1998). O BBT como instrumento diagnóstico em orientação profissional: uma abordagem psicodinâmica. Revista da ABOP, 2(1), 59-76.

Melo-Silva, L. L (1999). Pesquisas brasileiras no domínio da orientação vocacional e profissional. Disponível em: $<$ http://www.aiospconference2005.pt/abstracts/178.pd f>. Acesso em: março/2006.

Mignolet, L. (1960). O problema da senilidade e a orientação profissional. Arquivos Brasileiros de Psicotécnica, 12 (4), 107-114.

Moura, C. B. \& Menezes, M. V. (2004). Mudando de opinião: análise de um grupo de pessoas em condição de re-escolha profissional. Revista Brasileira de Orientação Profissional, 5(1), 29-45.

Moura, C. B., Sampaio, A. C. P., Menezes, M. V. \& Rodrigues, L. D. (2003) Uso de relatos escritos como instrumento de avaliação e intervenção em orientação profissional. Estudos de psicologia, 20 (3), 83-98.

Moura, C. B.; Silveira, J. M. (2002). Orientação profissional sob o enfoque da análise do comportamento: avaliação de uma experiência. Estudos de Psicologia, 19(1), 514.

Nascimento, R. S. G. F. (2004). Adolescência, identidade e escolha profissional: relato de uma experiência e seu referencial teórico. Psicologia Revista, 13(1), 31-39.

Nickel, D. C. (1996). O papel do orientador profissional. Psicologia Argumento, 14 (19), 151-155.
Noronha, A. P. P., Freitas, F. A., Ottati, F. (2003), Análise de instrumentos de avaliação de interesses profissionais. Psicologia Teoria e Pesquisa, 19 (3), 287-291.

Okino, E. T. K., Noce, M. A., Assoni, R. F., Corlatti, C. T \& Jacquemin, A. (2003). A adaptação do BBT Teste de Fotos de Profissões - para o contexto sociocultural brasileiro. Revista Brasileira de Orientação Profissional, 4 (1/2), 87-96.

Oliveira, I. D. (1999). A formação do orientador profissional e as mudanças atuais. Revista da ABOP, 3(1), 77 84.

Oliveira, M. B. L. (1995). Programa de intervenção em orientação profissional com um grupo experimental. Doxa, 1 (2), 89-108.

Ottati, F., Noronha, A. P. P. \& Salviati, M. (2003). Testes psicológicos: qualidade de instrumentos de interesse profissional. Interação, 7 (1).

Pasqualini, J. C., Garbulho, N. F. \& Schut, T. (2004). Orientação profissional com crianças: uma contribuição à educação infantil. Revista Brasileira de Orientação Profissional, 5(1), 71-85.

Primi, R., Casellato, E. O. \& Moggi, M. A. (2005) Estudo correlacional entre habilidades, personalidade e dificuldade de escolha profissional. Manuscrito em preparação.

Primi, R., Moggi, M. A., \& Casellato, E. O. (2004) Estudo correlacional do inventário de busca auto-dirigida (self-directed search) com o IFP. Psicologia Escolar e Educacional, 8(1), 47-54.

Rapold, R. C. M. (2003). A Psicologia da Educação na Bahia: a história do Instituto de Orientação Vocacional - IDOV - pela memória de seus personagens. Psicologia Educacional, 17, 95-118.

Ribeiro, C. de C. \& Bessa, N. M. (1960). Estudo das preferências de um grupo de orientandos do colégio Nova Friburgo. Arquivos Brasileiros de Psicotécnica, 12 (2), 23-30.

Santos, O. B. (1966). Psicologia aplicada aos problemas de colocação profissional de cegos no SENAI. Boletim de psicologia, 18/19 (51/54).

Santos, O. B. (1967) Psicologia aplicada aos problemas de colocação profissional de cegos no SENAI. Boletim de Psicologia, 18/19 (51/54).

Scheefer, R. (1973). Dois aspectos do comportamento vocacional: escolha e maturidade. Arquivos Brasileiros de Psicologia Aplicada, 25 (4), 5-14.

Silva, A. R. (1966). Confirmação da escolha profissional. Arquivos Brasileiros de Psicotécnica, 18(4), 101-104. 
Silva, L. L. M., Jacquemin, A. (2000). Contribuição para a interpretação do BBT de Martin Achtnich: a história das cinco fotos preferidas. Psic, 1(3), 72-79.

Silva, M. B. (1999). A formação do orientador profissional. Revista da ABOP, 3(1), 161-165.

Silva, M. B. (2001). Re-Orientação: redefinição da escolha profissional. Psicologia argumento, 19(28), 13-16.

Soares-Lucchiari, D. H. (1997). A re-orientação: apoio e época de crise. Revista da ABOP, 1(1), 81-88.

Sparta, M. (2003). O desenvolvimento da orientação profissional no Brasil. Revista Brasileira de Orientação Profissional, 4(1/2), 1-11.
Standards for educational and psychological testing. (1985). Washington, DC: American Psychological Association.

Velloso, R. (2000). O que há de psicanalítico em um acompanhamento vocacional. Cógito, 2, 69-74.

Witter, G. P. (1999). Metaciência e leitura. Em G. P. Witter (Org.). Leitura: Textos e Pesquisas (pp. 13 -22). Campinas: Editora Alínea.
Recebido em abril de 2006 Aprovado em junho de 2006

Sobre os autores:

Ana Paula Porto Noronha é doutora em Psicologia: Ciência e Profissão pela Pontifícia Universidade Católica de Campinas, docente do Programa de Pós-graduação Stricto Sensu em Psicologia da Universidade São Francisco e bolsista Produtividade em Pesquisa do CNPq.

Rodolfo Augusto Matteo Ambiel é graduando em Psicologia pela Universidade São Francisco e bolsista de Iniciação Cientíca PIBIC/CNPq. 